\title{
AlocaÇÃo de Fígados PARA TRANSPLANTE EM ADULTOS: VANTAGENS E DESVANTAGENS DO ESCORE MELD
}

\author{
LIVER TRANSPLANT ALLOCATION IN ADULTS: ADVANTAGES AND \\ DISADVANTAGES OF THE MELD SCORE
}

\author{
Guilherme Mariante-Neto ${ }^{1}$, Alfeu de Medeiros Fleck Júnior², \\ Ajácio Bandeira de Mello Brandão ${ }^{1,2}$
}

Clin Biomed Res. 2014;34(4):342-346

1 Programa de Pós-Graduação em Medicina: Hepatologia, Universidade Federal de Ciências da Saúde de Porto Alegre (UFCSPA). Porto Alegre, RS, Brasil.

2 Grupo de Transplante Hepático, Complexo Hospitalar Santa Casa de Misericórdia de Porto Alegre. Porto Alegre, RS, Brasil.

Autor correspondente:

Ajácio Bandeira de Mello Brandão

E-mail: ajaccio@via-rs.net

Grupo de Transplante Hepático

Complexo Hospitalar Santa Casa de

Misericórdia de Porto Alegre

Rua Professor Annes Dias, 295.

90020-090, Porto Alegre, RS, Brasil.

\section{RESUMO}

O processo de alocação de enxertos para transplante hepático é muito complexo em razão, principalmente, da discrepância entre o número de candidatos e o de doadores. O Model for End-Stage Liver Disease (MELD) é um escore de gravidade, desenvolvido nos Estados Unidos, que constitui um robusto preditor de sobrevida de pacientes em lista de espera para transplante hepático. Desde 2006, o Brasil adota o escore MELD para ordenar os receptores em uma fila de espera, com a política de atender antes o mais doente. Sua adoção como critério de alocação reduziu o número de óbitos em lista sem comprometer os resultados do transplante. Há situações que não são bem "atendidas" pelo MELD porque, ou a gravidade da situação clínica independe do grau da hepatopatia, ou o risco de permanecer em lista não é a morte, mas sim a doença avançar além de um ponto em que o transplante não possa ser realizado. Nesses casos, considerados "especiais", os pacientes recebem pontuação diferenciada no escore, com o intuito de transplantá-los em tempo hábil. Há estudos com o objetivo de aperfeiçoar o MELD, mantendo sempre a objetividade e transparência do escore original.

Palavras-chave: MELD score; transplante hepático

\section{ABSTRACT}

The process of graft allocation for liver transplant is very complex, especially due to the discrepancy between the number of transplant candidates and of donors. The Model for End-Stage Liver Disease (MELD) is a severity score developed in the United States that constitutes a strong survival predictor for patients on the waiting list for liver transplantation. Since 2006, Brazil has been using the MELD score to rank transplant candidates on a waiting line, with the policy of treating the sickest first. The implementation of this score as the allocation criterion reduced the number of deaths on the waiting list without compromising transplant outcomes. However, some situations are not well "treated" by the MELD score because either the severity of the clinical situation does not depend on the degree of liver disease or the risk of remaining on the list is not death but rather disease progression to a point that makes the transplant not feasible. In these so-called "special" cases, patients are graded differently on the MELD score, with the purpose of performing their transplantation in a timely manner. Studies have been conducted aiming to improve the MELD score while keeping the objectivity and transparency of the original score.

Keywords: MELD score; liver transplantation 
O transplante hepático $(\mathrm{TxH})$ oferece uma potencial cura para pacientes com doenças hepáticas terminais. Uma vez indicado e excluídas contraindicações, sua realização é ainda assim incerta, pois depende de um órgão disponível. Em outras palavras, a despeito de serem candidatos adequados ao procedimento, alguns pacientes não realizarão o TxH e evoluirão ao óbito em lista de espera. Como as taxas de doação, ao longo dos anos, têm sido consideravelmente menores que as necessárias, esse fato, sem políticas oficiais efetivas, tende a se agravar ${ }^{1}$. Nesse contexto, estabelecer critérios para a distribuição de órgãos escassos é um desafio: devemos priorizar os pacientes que mais precisam sem perder de vista os melhores desfechos possíveis. Então, um processo de alocação justo, apropriado e transparente é prérequisito indispensável tanto para os candidatos como para os doadores ${ }^{2,3}$.

O Brasil possui um programa de transplantes já consolidado. Desde 2006, houve aumento progressivo nas taxas de doadores efetivos, atingindo, em 2012, a taxa de 12,6 por milhão de população (pmp). Países como Espanha e Estados Unidos (EUA), no mesmo período, apresentaram taxas bem mais expressivas de doadores efetivos: 35,1 e 25,6, respectivamente. Especificamente em relação ao TxH, em 2012 foram realizados, em nosso país, 1.712 procedimentos (ou seja, 9,0 pmp). Assim, entre 30 países, atingimos o segundo lugar em número absoluto de $\mathrm{TxH}$ realizados. No mesmo período, Espanha e EUA realizaram 1.084 e 5.134 transplantes hepáticos, que correspondem a 23,2 e 16,1 procedimentos pmp, respectivamente. Portanto, com mais de 190 milhões de habitantes, atendemos cerca de $30 \%$ da necessidade anual para transplantes hepáticos ${ }^{4}$.

Nos EUA, a United Network for Organ Sharing (UNOS), instituição que administra e regula todos os assuntos relacionados com transplantes, há muitos anos reconheceu a necessidade de incorporar alguma medida de gravidade da doença hepática na alocação de enxertos ${ }^{5}$. Inicialmente, a política de alocação categorizava os pacientes em quatro grupos, de acordo com a gravidade do caso (sem uma definição clara do que se entendia por gravidade) e o local em que estavam sendo atendidos: status 1 (pacientes com insuficiência hepática aguda grave ou não funcionamento primário do enxerto, categorizados como necessitando de um enxerto com urgência máxima), status 2 (pacientes internados em unidades de tratamento intensivo), status 3 (pacientes hospitalizados) e status 4 (pacientes aguardando o TxH no domicílio) 5 . Ficou claro que tal sistema era dependente das práticas e comportamentos dos médicos assistentes e não refletia, necessariamente, a gravidade do caso em questão ${ }^{5}$. Para reforçar o papel da gravidade da doença hepática na política de alocação, posteriormente a UNOS adotou o escore Child-Turcotte-Pugh (CTP) ${ }^{6}$, muito utilizado pelos médicos para estimar a gravidade e o prognóstico de doenças hepáticas crônicas ${ }^{5,7}$. Contudo, entre outros óbices, o CTP não foi validado como ferramenta para predizer óbito em lista de espera para $\mathrm{TxH}$, seus componentes e pontos de corte foram selecionados empiricamente, e suas variáveis clínicas (ascite e encefalopatia hepática) são julgadas de maneira subjetiva. Como o escore agrupa os pacientes com doenças hepáticas crônicas em apenas três categorias $(A, B, C)$ havia um acúmulo dos casos mais graves na categoria $C$, assim que o tempo em lista de espera passou a ser usado como desempate. Portanto, a utilização do escore CTP na alocação de enxertos também passou a ser questionada, entre outros aspectos, devido às altas taxas de mortalidade de pacientes em lista de espera para $\mathrm{TxH}$ com enxerto proveniente de doador falecido.

\section{O escore MELD (Model for End-stage Liver Disease)}

Em 2000, foi publicado um modelo matemático desenvolvido para predizer o risco de morte em três meses de cirróticos adultos com hipertensão portal que necessitavam submeter-se a anastomose portossistêmica intra-hepática transjugular ou TIPS (do inglês Transjugular Intrahepatic Portosystemic Shunt) $)^{8}$. O escore original incluía três parâmetros laboratoriais (creatinina sérica, bilirrubina total e tempo de protrombina, expresso como INR [international normalized ratio]) e um parâmetro clínico (etiologia da cirrose $)^{8}$. Estudo posterior ${ }^{9}$ mostrou que a acurácia do modelo não era alterada com a supressão da variável "etiologia da cirrose", assim que o modelo ficou apenas com variáveis objetivas. A acurácia do MELD em estimar a sobrevida de cirróticos em lista de espera para TxH ou não foi demonstrada em vários centros ${ }^{10,11}$, inclusive em nosso meio ${ }^{12}$. Os resultados desses estudos mostraram valores da estatística-c próximos ou mesmo superiores a 0,8 , refletindo um valor preditivo de boa significância clínica. Em outras palavras: um valor de 0,8 indica que, quando um par de pacientes é aleatoriamente retirado da população em estudo, o escore irá predizer corretamente, em $80 \%$ das vezes, qual paciente morrerá antes ${ }^{9,13}$. A acurácia do escore MELD em estimar a sobrevida de pacientes em lista de espera para $\mathrm{TxH}$ também foi mostrada. Portanto, o MELD tem vários aspectos que o tornam um modelo prognóstico de sobrevida muito interessante para uso em alocação de enxertos em candidatos a $\mathrm{TxH}:$ a) incorpora apenas variáveis objetivas e 
de simples obtenção, tendo cada variável um peso proporcional à sua influência no prognóstico; b) é independente da etiologia da cirrose; c) possui alto poder preditivo; d) a gravidade da doença hepática é classificada de forma contínua, ao contrário do efeito "teto" do CTP.

\section{Escore MELD na alocação de enxertos para transplante hepático nos EUA}

Dentro da política de "atender antes o mais doente", a UNOS adotou o escore MELD para alocação de fígados para adultos em fevereiro de 2002. Nesse contexto, o cálculo do escore foi alterado levemente, fixando o valor mínimo de 1,0 para cada variável para evitar escores negativos e, adicionalmente, fixando em 4,0 o valor máximo da creatinina, incluindo pacientes em hemodiálise. Os aplicativos disponíveis calculam o MELD empregando as modificações efetuadas pela UNOS, sendo que o escore varia de 6-40 e valores mais altos representam maior risco de morte ${ }^{14}$. Estudos com grandes bases de dados apontaram que a introdução do MELD na alocação de enxertos de doadores falecidos para TxH em adultos atingiu o objetivo planejado: reduzir o número de óbitos em lista sem comprometer os resultados do procedimento ${ }^{15-18}$. Entretanto, também mostraram que pacientes com escores mais altos no transplante (com diferentes pontos de corte) tinham maiores taxas de mortalidade no pós-operatório ${ }^{17,19}$.

\section{O benefício do transplante hepático de acordo com o MELD}

Estudo norte-americano ${ }^{20}$ avaliou o benefício do $\mathrm{TxH}$ comparando pacientes transplantados com candidatos comparáveis não transplantados. A conclusão foi a de que o benefício do TxH é maior em pacientes com escores mais altos (portanto, com maior risco de morte). Por exemplo, para pacientes com MELD entre 18-20, o risco de morte foi $38 \%$ menor $(P<0,01)$ entre os transplantados em comparação com os candidatos; o benefício aumentou à medida que aumentou o escore, até um máximo de 40 , quando o risco de morte foi $96 \%$ menor nos transplantados, em comparação com os candidatos. O estudo também mostrou que, em pacientes com escores baixos, o TxH aparentemente não tem benefício (considerando seguimento de 1 ano). Assim, o risco de morte foi 3,6 vezes maior nos transplantados com MELD 11 em comparação aos pacientes com o mesmo escore e que permaneceram em lista (hazard ratio 3,6; $\mathrm{P}<0.01)$. O benefício do $\mathrm{TxH}$ e sua correlação com o escore MELD foi validado em nosso meio ${ }^{21}$.

\section{Escore MELD na alocação de enxertos para}

\section{transplante hepático no Brasil}

Até 2006, no Brasil, o critério de alocação de enxertos de doadores falecidos para pacientes com doenças hepáticas crônicas considerava, além da compatibilidade $\mathrm{ABO}$, a data da inscrição em lista de espera, ou seja, era estritamente cronológico, dentro da filosofia "atender primeiro quem foi listado primeiro". Atendendo às demandas de vários grupos transplantadores contrários a esse critério, em maio daquele ano o Sistema Nacional de Transplantes mudou radicalmente sua conduta e adotou o critério de "antes, o mais doente", sendo a gravidade da doença hepática estimada através do escore MELD e utilizando-se para seu cálculo a mesma fórmula adotada pela UNOS ${ }^{22}$.

Em nosso país não existe uma base de dados com os resultados nacionais; assim, o impacto da introdução do MELD na alocação de enxertos foi avaliado em alguns centros isolados ${ }^{23,24}$, o que não é o ideal, mas o possível. Os resultados também evidenciaram uma redução da mortalidade em lista de espera sem comprometimento relevante dos resultados. Portanto, os objetivos principais da adoção do MELD na alocação de enxertos foram atingidos.

\section{Pontos fracos do MELD na alocação de enxer- tos}

Há situações que não são bem "atendidas" pelo MELD porque, ou a gravidade da situação clínica independe do grau da hepatopatia, ou o risco de permanecer em lista não é a morte, mas sim a doença avançar além de um ponto em que o transplante não possa ser realizado ${ }^{25}$. Nesses casos, considerados "especiais", os pacientes recebem pontuação diferenciada no escore, com o intuito de transplantá-los em tempo hábil. De acordo com a legislação vigente ${ }^{22}$, são consideradas situações especiais:

- Tumor neuroendócrino metastático, irressecável, com tumor primário já retirado, e sem doença extra-hepática detectável;

- Carcinoma hepatocelular dentro dos critérios de Milão e com contraindicação de ressecção;

- Polineuropatia amiloidótica familiar - graus I e II;

- Síndrome hepatopulmonar - $\mathrm{PaO}_{2}$ menor que $60 \mathrm{~mm} / \mathrm{Hg}$ em ar ambiente;

- Hemangioma gigante irressecável com síndrome compartimental, adenomatose múltipla, hemangiomatose ou doença policística; 
- Carcinoma fibrolamelar irressecável e sem doença extra-hepática;

- Adenomatose múltipla irressecável com presença de complicações;

- Doenças metabólicas com indicação de transplante (por exemplo, fibrose cística, doença policística, deficiência de alfa-1antitripsina, doença de Wilson, oxalose primária).

É importante considerar que a pontuação adicional atribuída aos pacientes foi criada, de certa maneira, arbitrariamente, o que é inadequado. A situação especial mais comum, em todo o mundo, é o carcinoma hepatocelular ${ }^{25}$.

Certos casos, contudo, não se encaixam no rol de situações especiais, como, por exemplo, pacientes com provas de função hepática pouco alteradas e com complicações clínicas graves ou incapacitantes, como ascite intratável e encefalopatia hepática crônica. Para esses casos, foram criados protocolos, muito rígidos, que devem ser preenchidos pelos médicos transplantadores e submetidos à análise de Câmara Técnica ${ }^{22}$. Caso a solicitação seja aceita, os pacientes serão priorizados em lista de espera.

\section{CONCLUSÕES}

O sistema de alocação baseado no escore MELD é transparente e centrado no paciente. Sua utilização na alocação de enxertos provenientes de doadores falecidos trouxe importantes vantagens em relação ao antigo sistema de alocação, e os períodos que precedem e seguem a sua implantação são denominados de eras pré e pós MELD. Contudo, o escore - como previsto - não é perfeito e há muitos estudos objetivando melhorar e refinar a fórmula original. Contudo, todas as propostas de mudança continuam baseadas no escore MELD original.

\section{Conflito de interesse}

Os autores não têm conflitos de interesse a divulgar.

\section{REFERÊNCIAS}

1. Marroni CA, Fleck AM JR, Brandão ABM. Indicações e contraindicações do transplante hepático. In: Mattos AA, Dantas-Corrêa EB, editores. Tratado de hepatologia. Rio de Janeiro: Rubio; 2010. p. 877-89.

2. Bobbert M, Ganten TM. Liver allocation: urgency of need or prospect of success? Ethical considerations. Clin Transplant. 2013;27(Suppl 25):34-9. http://dx.doi. org/10.1111/ctr.12154. PMid:23909500

3. Durand $F$. The quest for equity in liver transplantation: another lesson learned from women. J Hepatol. 2011;54(3):401-2. http://dx.doi. org/10.1016/j.jhep.2010.09.001. PMid:21126789

4. Associação Brasileira de Transplantes de Órgãos - ABTO. Registro Brasileiro de Transplantes. 2013;4:1-85.

5. Freeman RB JR. The model for endstage liver disease comes of age. Clin Liver Dis. 2007;11(2):249-63. http:// dx.doi.org/10.1016/j.cld.2007.04.005. PMid:17606205

6. Pugh RN, Murray-Lyon IM, Dawson JL, Pietroni MC, Williams R. Transection of the oesophagus for bleeding oesophageal varices. $\mathrm{Br}$
J Surg. 1973;60(8):646-9. http:// dx.doi.org/10.1002/bjs.1800600817. PMid:4541913

7. Merion RM. Current status and future of liver transplantation. Semin Liver Dis. 2010;30(4):411-21. http:// dx.doi.org/10.1055/s-0030-1267541. PMid:20960380

8. Malinchoc M, Kamath PS, Gordon FD, Peine CJ, Rank J, ter Borg PC. A model to predict poor survival in patients undergoing transjugular intrahepatic portosystemic shunts. Hepatology. 2000;31(4):864-71. http:// dx.doi.org/10.1053/he.2000.5852. PMid:10733541

9. Kamath PS, Wiesner RH, Malinchoc M, Kremers W, Therneau TM, Kosberg $\mathrm{CL}$, et al. A model to predict survival in patients with end-stage liver disease. Hepatology. 2001;33(2):464-70. http:// dx.doi.org/10.1053/jhep.2001.22172. PMid:11172350

10. Said A, Williams J, Holden J, Remington P, Gangnon R, Musat A, et al. Model for end stage liver disease score predicts mortality across a broad spectrum of liver disease. J Hepatol. 2004;40(6):897903. http://dx.doi.org/10.1016/j. jhep.2004.02.010. PMid:15158328
11. Botta F, Giannini E, Romagnoli $P$, Fasoli A, Malfatti F, Chiarbonello $B$, et al. MELD scoring system is useful for predicting prognosis in patients with liver cirrhosis and is correlated with residual liver function: a European study. Gut. 2003;52(1):134-9. http:// dx.doi.org/10.1136/gut.52.1.134. PMid:12477775

12. Brandão A, Fuchs SC, Gleisner AL, Marroni C, Zanotelli ML, Cantisani G. Model for the end-stage liver disease and death prediction in a cohort of Brazilian patients on the waiting list for liver transplantation. Clin Transplant. 2008;22(5):651-6. http://dx.doi.org/10.1111/j.13990012.2008.00860.x. PMid:18549449

13. Zou KH, O'Malley AJ, Mauri L. Receiver-operating characteristic analysis for evaluating diagnostic tests and predictive models. Circulation. 2007;115(5):6547. http://dx.doi.org/10.1161/ CIRCULATIONAHA.105.594929. PMid:17283280

14. Freeman RB JR, Wiesner RH, Harper A, McDiarmid SV, Lake J, Edwards E, et al. The new liver allocation system: moving toward evidencebased transplantation policy. Liver 
Transpl. 2002;8(9):851-8. http:// dx.doi.org/10.1053/jts.2002.35927. PMid:12200791

15. Freeman RB, Wiesner RH, Edwards E, Harper A, Merion R, Wolfe R, et al. Results of the first year of the new liver allocation plan. Liver Transpl. 2004;10(1):7-15. http://dx.doi. org/10.1002/lt.20024. PMid:14755772

16. Kanwal F, Dulai GS, Spiegel BM, Yee HF, Gralnek IM. A comparison of liver transplantation outcomes in the pre- vs. post-MELD eras. Aliment Pharmacol Ther. 2005;21(2):169-77. http://dx.doi.org/10.1111/j.13652036.2005.02321.x. PMid:15679767

17. Yoo HY, Thuluvath PJ. Short-term postliver transplant survival after the introduction of MELD scores for organ allocation in the United States. Liver Int. 2005;25(3):536-41. http://dx.doi.org/10.1111/j.14783231.2005.01011.x. PMid:15910490

18. Berg CL, Steffick DE, Edwards EB, Heimbach JK, Magee JC, Washburn WK, et al. Liver and intestine transplantation in the United States 1998-2007. Am J Transplant. 2009;9(4 Pt 2):907-31. http://dx.doi.org/10.1111/ j.1600-6143.2009.02567.x.

\section{PMid:19341415}

19. Freeman RB, Harper A, Edwards EB. Excellent liver transplant survival rates under the MELD/PELD system. Transplant Proc. 2005;37(2):5858. http://dx.doi.org/10.1016/j. transproceed.2004.12.099. PMid:15848465

20. Merion RM, Schaubel DE, Dykstra DM, Freeman RB, Port FK, Wolfe RA. The survival benefit of liver transplantation. $A m$ J Transplant. 2005;5(2):307-13. http://dx.doi.org/10.1111/j.16006143.2004.00703.x. PMid:15643990

21. Gleisner AL, Muñoz A, Brandao A, Marroni C, Zanotelli ML, Cantisani GG, et al. Survival benefit of liver transplantation and the effect of underlying liver disease. Surgery. 2010;147(3):392-404. http://dx.doi. org/10.1016/j.surg.2009.10.006. PMid:19962165

22. Brasil. Ministério da Saúde. Portaria $n^{\circ} 1.160$ de 29 de maio de 2006. Modifica os critérios de distribuição de fígado de doadores cadáveres para transplante, implantando o critério de gravidade de estado clínico do paciente. Diário Oficial da União
[Internet]. 2006 Maio 31 [capturado em 2014 Jul 12]. Disponível em: http://dtr2001.saude.gov.br/sas/ PORTARIAS/Port2006/GM/GM-1160. htm

23. da Silva Machado AG, de Medeiros Fleck A JR, Marroni C, Zanotelli ML, Cantisani G, de Mello Brandão AB. Impact of MELD score implementation on liver allocation: experience at a Brazilian center. Ann Hepatol. 2013;12(3):440-7. PMid:23619261.

24. Pestana RC, Baracat EI, Massarollo PC, Pereira LA, Szutan LA. Consequences of the implementation of the Model for End-stage Liver Disease system for liver allocation in Brazil. Transplant Proc. 2013;45(6):21114. http://dx.doi.org/10.1016/j. transproceed.2012.11.007. PMid:23747144

25. Northup PG, Intagliata NM, Shah NL, Pelletier SJ, Berg CL, Argo CK. Excess mortality on the liver transplant waiting list: Unintended policy consequences and model for End-Stage Liver Disease (MELD) inflation. Hepatology. 2015;61(1):28591. http://dx.doi.org/10.1002/ hep.27283. PMid:24995689

Recebido: 14/07/2014 Aceito: 03/08/2014 